\title{
Draft Genome Sequence Resource of Switchgrass Rust Pathogen, Puccinia novopanici Isolate Ard-01
}

Upinder S. Gill, Raja Sekhar Nandety, Nick Krom, Xinbin Dai, Zhaohong Zhuang, Yuhong Tang, Patrick X. Zhao, and Kirankumar S. Mysore ${ }^{\dagger}$

Noble Research Institute, LLC., Ardmore, OK, 73401, U.S.A.

\begin{abstract}
Puccinia novopanici is an important biotrophic fungal pathogen that causes rust disease in switchgrass. Lack of genomic resources for $P$. novopanici has hampered the progress toward developing effective disease resistance against this pathogen. Therefore, we have sequenced the whole genome of $P$. novopanici and generated a framework to understand pathogenicity mechanisms and identify effectors, repeat element invasion, genome evolution, and comparative genomics among Puccinia spp. in the future. Long- and short-read sequences were generated from $P$. novopanici genomic DNA by PacBio and Illumina technologies, respectively, and assembled a 99.9-Mb genome. Transcripts of $P$. novopanici were predicted from assembled genome using MAKER and were further validated by RNAseq data. The genome sequence information of $P$. novopanici will be a valuable resource for researchers working on monocot rusts and plant disease resistance in general.
\end{abstract}

\section{Genome Announcement}

Switchgrass rust caused by Puccinia novopanici is a significant disease of switchgrass (Panicum virgatum L.), an important biofuel and forage crop in the United States of America. Severe infestations of switchgrass rust under suitable environmental conditions have been reported in Oklahoma during past several years (Uppalapati et al. 2013). Puccinia novopanici was mistakenly reported as $P$. emaculata in previous publications (Demers et al. 2017; Gill et al. 2015, 2018; Uppalapati et al. 2013). However, sequence comparison of our fungal isolates with suggested $P$. novopanici sequences in recent reports (Kenaley et al. 2016; Orquera-Tornakian et al. 2017) confirmed our isolates as $P$. novopanici. $P$. novopanici is an obligate biotrophic fungal pathogen and has a mode of infection similar to other Puccinia spp. such as wheat and barley rust pathogens (Gill et al. 2015; Uppalapati et al. 2013). Variations in the degree of virulence in $P$. novopanici (reported as $P$. emaculata) have been previously shown in the United States (Frazier et al. 2013; Hirsch et al. 2010; Kenaley et al. 2016; Li et al. 2009; Uppalapati et al. 2013; Zale et al. 2008). These variations among isolates can be explained by differences at the genomic level. A recent population study using simple sequence repeat markers also suggested genomic differences among isolates collected from different locations in the United States (Orquera-Tornakian et al. 2017). Such heterogeneity in $P$. novopanici populations poses a risk to switchgrass cultivation due to development of new

\section{U. S. Gill and R. S. Nandety contributed equally to this work.}

${ }^{\dagger}$ Corresponding author: K. S. Mysore; ksmysore@ noble.org

Current address of U. S. Gill: Gulf Coast Research and Education Center, University of Florida, Wimauma, FL 33598, U.S.A.

*The e-Xtra logo stands for "electronic extra" and indicates that one supplementary table is published online.

The author(s) declare no conflict of interest.

Accepted for publication 1 May 2019.
Funding

This work was supported by Noble Research Institute, LLC and, in part, by National Science Foundation's Experimental Program to Stimulate Competitive Research (EPS-0814361).

\section{Keywords}

mycology 
Table 1. Sequence data of genome and transcriptome of Puccinia novopanici isolate Ard-01

\begin{tabular}{lc} 
Parameters & Results \\
Genome size & $99,934,463 \mathrm{bp}$ \\
Number of contigs & 11,088 \\
Minimum contig length & $2,034 \mathrm{bp}$ \\
Maximum contig length & $7,332 \mathrm{bp}$ \\
N50 contig length & $13,091 \mathrm{bp}$ \\
N90 contig length & $4,215 \mathrm{bp}$ \\
Mean contig length & $9,164 \mathrm{bp}$ \\
GC content & $44.9 \%$ \\
Transcripts & 16,622 \\
\hline
\end{tabular}

virulent races of the pathogen via recombination and mutagenesis. Therefore, efforts in our laboratory are directed toward understanding the pathogen biology and diversity via wholegenome sequencing and comparative genomics with other sequenced and well-studied Puccinia genomes: $P$. triticina, $P$. striiformis f. sp. tritici, $P$. graminis f. sp. tritici, and $P$. sorghi.

For this study, $P$. novopanici isolates were collected from switchgrass plants grown in Ardmore, OK in the United States and confirmed by nuclear ribosomal DNA internal transcribed spacer (nuclear rDNA ITS) region amplification (Uppalapati et al. 2013). For wholegenome sequencing, urediniospores from isolate Ard-01 were inoculated on switchgrass cultivar Summer and a single pustule was selected for further rounds of inoculations on fresh, infection-free switchgrass plants to multiply urediniospores. All inoculations were performed in clean growth chambers at day and night temperatures of 29 and $22^{\circ} \mathrm{C}$, respectively, with a 16-h photoperiod, $90 \%$ relative humidity, and photon flux density of 150 to $200 \mu \mathrm{mol} \mathrm{m} \mathrm{m}^{-2}$. For genomic DNA isolation, urediniospores were germinated in germination solution, as described previously (Gill et al. 2018). Genomic DNA was isolated by a Quick-DNA Fungal/ Bacterial Kit (Zymo Research). PacBio (Pacific Biosciences) and Illumina (Illumina Inc.) chemistries were used to generate long- and short-read sequences, respectively, and to generate hybrid assembly. A PacBio RS II library was constructed with an average insert size of $20 \mathrm{~kb}$ and sequenced using P4-C2 chemistry to generate $21,880,460,804$ bp of data ( 22 $\mathrm{GB})$, achieving $215 \times$ coverage for the genome. For Illumina sequencing, $2 \times 300$-bp reads were generated from inserts of $550 \mathrm{bp}$ using Illumina MiSeq sequencer. PacBio and Illumina reads were assembled separately by using Canu (Koren et al. 2017) and Masurca (Zimin et al. 2013), respectively. De novo assembly of the processed data were performed using the Hybrid assembly method using CLC Genomics Workbench 11.0 (https:// www.qiagenbioinformatics.com/). Genome assembly and transcript prediction results are presented in Table 1. The whole-genome assembly of $P$. novopanici resulted in a total genome size of $99.934 \mathrm{Mb}$ assembled into 11,088 super scaffolding contigs, which is similar in size to its closest relative, P. sorghi, with genome size of 99.6 Mb (Rochi et al. 2018). Based on the Assemblathon (http://assemblathon.org/) statistics of the $P$. novopanici assembled genome, there were 11,088 predicted contigs in $P$. novopanici with average length of $9,164 \mathrm{bp}$. The assembled contigs are in line with the assemblies reported by Cantu et al. (2011) and Cuomo et al. (2017). The average N50 scaffold length was 13,091 bp, with shortest and longest scaffolds being 2,034 and 73,332 bp, respectively. The $P$. novopanici genome assembly was annotated using MAKER (Campbell et al. 2014; Cantarel et al. 2008). The input resource of MAKER comprised an in-house database consisting of six Illumina RNA-seq samples representing $P$. novopanici treated with different concentrations of phosphite (Gill et al. 2018). In addition, the protein sequences from the Uniprot database (https://www.uniprot.org/) and Joint Genome Institute phytozome (https://phytozome.jgi. doe.gov/pz/portal.html) annotation of $P$. graminis, $P$. striiformis, and $P$. triticina were included as input for MAKER. Repeat Mask (http://repeatmasker.org) was applied to remove retrotransposon sequences and low-complexity regions in the genome during gene model prediction in MAKER (Muszewska et al. 2011; Soliai et al. 2014). The MAKER pipeline was iteratively run three times to generate optimized 19,064-gene models. A final filtration step was performed to remove redundant duplicated gene models that resulted in 16,622 transcripts. Genome annotation was supported and further validated by available $P$. novopanici RNAseq data (Gill et al. 2018) wherein 76 to $81 \%$ of RNAseq reads were mapped to the finished hybrid $P$. novopanici assembly. Furthermore, the annotated contigs based on the RNAseq studies correlated with the predicted genes from genome sequence analysis. Blast 
results of $P$. novopanicitranscripts identified the maximum number of best hits from $P$. sorghi (76.1\%) followed by $P$. graminis f. sp. tritici (15\%) and $P$. striiformis f. sp. tritici $(7.2 \%)$. Previous phylogenetic studies based on rDNA ITS sequences suggested $P$. asparagi as the closest relative of $P$. novopanici (Uppalapati et al. 2013). However, based on the assembled genome size and estimated homology, $P$. novopanici is relatively closer to $P$. sorghi (Rochi et al. 2018). Switchgrass, sorghum, and maize are C4 plants, hence suggesting more relatedness of $P$. novopanici with rust pathogens of $\mathrm{C} 4$ plants rather than $\mathrm{C} 3$ plants such as asparagus, wheat, and barley. Further investigations in the future by comparative genomics will reveal the diversity among these Puccinia spp.

The genome sequence of $P$. novopanici will provide an important resource for researchers working on $P$. novopanici detection, epidemiology, population genetics, and disease resistance. Comparative genomics with other worldwide economically important pathogens such as $P$. graminis f. sp. tritici that causes wheat stem rust and its closest relative based on the wholegenome sequence, $P$. sorghi, will help us better understand the evolution and diversity of Puccinia spp. in general. The sequence data of $P$. novopanici is available at the NCBI database under BioProject ID PRJNA507656; BioSample accession SAMN10502107. The raw data can be accessed at NCBI as SRA submissions (SRR8264864, SRR8264863, SRR8264866, SRR8264865, SRR8264868, and SRR8264867). Genome assembly of $P$. novopanici is made available at NCBI with the GenBank assembly accession number GCA_004348175.1. The annotated transcripts have been deposited at DDBJ/ENA/GenBank under the accession GHIU00000000. The version described in this article is the first version, GHIU01000000. In addition, the complete transcripts annotations along with the transcript IDs and the relevant NCBI accession numbers are presented in Supplementary Table S1.

\section{Acknowledgments}

We thank J. Gallaway for maintaining switchgrass plants in the greenhouse.

\section{Literature Cited}

Campbell, M. S., Holt, C., Moore, B., and Yandell, M. 2014. Genome annotation and curation using MAKER and MAKER-P. Curr. Protoc. Bioinf. 48:4.11.1-4. 11-39.

Cantarel, B. L., Korf, I., Robb, S. M., Parra, G., Ross, E., Moore, B., Holt, C., Sanchez Alvarado, A., and Yandell, M. 2008. MAKER: An easy-to-use annotation pipeline designed for emerging model organism genomes. Genome Res. 18:188-196.

Cantu, D., Govindarajulu, M., Kozik, A., Wang, M., Chen, X., Kojima, K. K., Jurka, J., Michelmore, R. W., and Dubcovsky, J. 2011. Next generation sequencing provides rapid access to the genome of Puccinia striiformis f. sp. tritici, the causal agent of wheat stripe rust. PLoS One 6:e24230.

Cuomo, C. A., Bakkeren, G., Khalil, H. B., Panwar, V., Joly, D., Linning, R., Sakthikumar, S., Song, X., Adiconis, X., Fan, L., Goldberg, J. M., Levin, J. Z., Young, S., Zeng, Q., Anikster, Y., Bruce, M., Wang, M., Yin, C., McCallum, B., Szabo, L. J., Hulbert, S., Chen, X., and Fellers, J. P. 2017. Comparative analysis highlights variable genome content of wheat rusts and divergence of the mating loci. G3: Genes Genomes Genet. 7:361-376.

Demers, J. E., Liu, M., Hambleton, S., and Castlebury, L. A. 2017. Rust fungi on Panicum. Mycologia 109:1-17.

Frazier, T., Shen, Z., Zhao, B., and Bush, E. 2013. First report of Puccinia emaculata infection on switchgrass in Virginia. Plant Dis. 97:424.

Gill, U. S., Sun, L., Rustgi, S., Tang, Y., von Wettstein, D., and Mysore, K. S. 2018. Transcriptome-based analyses of phosphite-mediated suppression of rust pathogens Puccinia emaculata and Phakopsora pachyrhizi and functional characterization of selected fungal target genes. Plant J. 93:894-904.

Gill, U. S., Uppalapati, S. R., Nakashima, J., and Mysore, K. S. 2015. Characterization of Brachypodium distachyon as a nonhost model against switchgrass rust pathogen Puccinia emaculata. BMC Plant Biol. 15:113.

Hirsch, R. L., TeBeest, D. O., Bluhm, B. H., and West, C. P. 2010. First report of rust caused by Puccinia emaculata on switchgrass in Arkansas. Plant Dis. 94:381.
Kenaley, S. C., Hudler, G. W., and Bergstrom, G. C. 2016. Detection and phylogenetic relationships of Puccinia emaculata and Uromyces graminicola (Pucciniales) on switchgrass in New York State using rDNA sequence information. Fungal Biol. 120:791-806.

Koren, S., Walenz, B. P., Berlin, K., Miller, J. R., Bergman, N. H., and Phillippy, A. M. 2017. Canu: Scalable and accurate long-read assembly via adaptive k-mer weighting and repeat separation. Genome Res. 27:722-736.

Li, Y., Windham, M., Trigiano, R., Windham, A., Ownley, B., Gwinn, K., Zale, J., and Spiers, J. 2009. Cultivar-specific interactions between switchgrass and Puccinia emaculata. (Abstr.) Phytopathology 99:S72.

Muszewska, A., Hoffman-Sommer, M., and Grynberg, M. 2011. LTR retrotransposons in fungi. PLoS One 6:e29425.

Orquera-Tornakian, G. K., Garrido, P., Kronmiller, B., Hunger, R., Tyler, B. M., Garzon, C. D., and Marek, S. M. 2017. Identification and characterization of simple sequence repeats (SSRs) for population studies of Puccinia novopanici. J. Microbiol. Methods 139:113-122.

Rochi, L., Dieguez, M. J., Burguener, G., Darino, M. A., Pergolesi, M. F., Ingala, L. R., Cuyeu, A. R., Turjanski, A., Kreff, E. D., and Sacco, F. 2018. Characterization and comparative analysis of the genome of Puccinia sorghi Schwein, the causal agent of maize common rust. Fungal Genet. Biol. 112:31-39.

Soliai, M. M., Meyer, S. E., Udall, J. A., Elzinga, D. E., Hermansen, R. A., Bodily, P. M., Hart, A. A., and Coleman, C. E. 2014. De novo genome assembly of the fungal plant pathogen Pyrenophora semeniperda. PLoS One 9:e87045.

Uppalapati, S. R., Serba, D. D., Ishiga, Y., Szabo, L. J., Mittal, S., Bhandari, H. S., Bouton, J. H., Mysore, K. S., and Saha, M. C. 2013. Characterization of the rust fungus, Puccinia emaculata, and evaluation of genetic variability for rust resistance in switchgrass populations. BioEnergy Res. 6:458-468.

Zale, J., Freshour, L., Agarwal, S., Sorochan, J., Ownley, B. H., Gwinn, K. D., and Castlebury, L. A. 2008. First report of rust on switchgrass (Panicum virgatum) caused by Puccinia emaculata in Tennessee. Plant Dis. 92:1710.

Zimin, A. V., Marcais, G., Puiu, D., Roberts, M., Salzberg, S. L., and Yorke, J. A. 2013. The MaSuRCA genome assembler. Bioinformatics 29:2669-2677. 